\title{
ALGEBRAIC COMBINATIONS OF EXPONENTIALS*
}

\author{
BY
}

J. F. RITT

By an exponential polynomial, we shall mean a function

$$
P(z)=a_{0} e^{\alpha_{0} z}+\cdots+a_{m} e^{\alpha_{m} z}
$$

with arbitrary complex numbers for $a^{\prime}$ 's and $\alpha$ 's. We shall refer to the $\alpha$ 's as the exponents of $P(z)$.

We study here functions defined by an equation

$$
P_{n} w^{n}+P_{n-1} w^{n-1}+\cdots+P_{0}=0,
$$

with every $P$ an exponential polynomial. When the exponents of the $P$ 's are all integers, the substitution $e^{z}=u$ converts $w$ into an algebraic function of $u$. Thus, in a sense, the theory of the equation (1) is a generalization of the theory of algebraic functions.

The present investigation arose out of the problem of determining all uniform functions which satisfy an equation (1). It is a natural conjecture that if $w$, defined by (1), is uniform, then $w=Q / R$ with $Q$ and $R$ exponential polynomials. One might, further, expect the exponents in $Q$ and $R$ to be linear combinations of the exponents in the $P$ 's, with rational coefficients. Both of these conjectures are verified by our work.

We have already proved $\dagger$ that if the quotient of two exponential polynomials is an integral function, the quotient is an exponential polynomial. This result is obtained again here, under the weaker hypothesis that the quotient is analytic in a sector of opening greater than $\pi$.

The problem of uniformity settled, we are able to discuss the relation between the reducibility properties of (1) and the number of analytic functions which (1) defines. Our result, which generalizes a well known theorem on algebraic functions, is stated in $\$ 21$.

In examining these questions, we were led to study the behavior, for large values of $z$, of functions determined by (1). It turns out that the Riemann surface for $w$ can be divided into a finite number of sectors, in each of which, after a border of the sector of finite width is removed, $w$ can be represented by a Dirichlet series with complex exponents. The sectors admit of precise description.

* Presented to the Society, February 23, 1929; received by the editors May 1, 1929.

$\dagger$ Ritt, (1). (List of references at end of paper.) 
In $\$ 21$ we prove that if $S$ is any sector of opening greater than $\pi$, the branch points of $w$ lying in $S$, and lying outside an arbitrarily large circle, serve, in their totality, to connect up all of the branches of $w$, and, in fact, to generate the entire group of monodromy of $w$.

The developability of $w$ in Dirichlet series with complex exponents is proved by the Newton polygon method of algebraic function theory, with appropriate modifications. We have found serviceable, also, the results of Tamarkin, Wilder and Pólya on the zeros of exponential polynomials, Hille's work on Dirichlet series with complex exponents, and the notion of almost periodic function.

\section{EquATIONS WHOSE COEFFICIENTS HAVE REAL EXPONENTS}

1. We consider an equation*

$$
f(w)=P_{n}(z) w^{n}+P_{n-1}(z) w^{n-1}+\cdots+P_{1}(z) w+P_{0}(z)=0,
$$

where each $P_{i}$ is a function defined by a Dirichlet series with a half-plane of absolute convergence

$$
P_{i}(z)=a_{i 1} e^{\lambda_{1} z}+a_{i 2} e^{\lambda_{2} z}+\cdots+a_{i p} e^{\lambda_{p} z}+\cdots,
$$

the $\lambda$ 's, independent of $i$, being real, and such that

$$
\lambda_{1}>\lambda_{2}>\cdots>\lambda_{p}>\cdots ; \lim _{p \rightarrow \infty} \lambda_{p}=-\infty .
$$

We make the non-restrictive assumption that $P_{0} \not \equiv 0, P_{n} \not \equiv 0$.

2. We shall prove that a real $A$ exists, such that, for $x>A \quad(z=x+$ $\left.y(-1)^{1 / 2}\right)$, the $n$ solutions $w_{1}, \cdots, w_{n}$ of (2) are analytic and representable by absolutely convergent Dirichlet series

$$
w_{i}(z)=c_{i 1} e^{\rho_{1} z}+c_{i 2} e^{\rho_{2} z}+\cdots+c_{i p} e^{\rho_{p} z}+\cdots,
$$

the $\rho$ 's, which decrease toward $-\infty$, having expressions

$$
\rho_{p}=\frac{1}{r}\left(m_{p 1} \lambda_{1}+m_{p 2} \lambda_{2}+\cdots+m_{p q_{p}} \lambda_{q_{p}}\right),
$$

with $r$ a positive integer independent of $p$, and with integral $m$ 's.

3. It will suffice to show that (2) has one solution of the form (3).

If $P_{i}(i=0, \cdots, n)$ is not identically zero, we represent by $\sigma_{i}$ the largest $\lambda$ which occurs in $P_{i}$ in a term with a coefficient $a$ not zero. If $P_{i} \equiv 0$, we let $\sigma_{i}=-\infty$.

\footnotetext{
* It is convenient to write $f(w)$ rather than $f(w, z)$.
} 
Let $g_{1}$ be the greatest value of $i(i=1, \cdots, n)$ which makes

$$
\frac{\sigma_{0}-\sigma_{i}}{i}
$$

a minimum. Let $\rho_{1}$ denote this minimum.

Let $\alpha_{i}{ }^{(1)}\left(i=1, \cdots, g_{1}\right)$ denote the coefficient of $e^{\sigma_{i z}}$ in $P_{i}$, or denote 0 , according as (5) equals $\rho_{1}$ or exceeds $\rho_{1}$. Let $\alpha_{0}{ }^{(1)}$ be the coefficient of $e^{\sigma_{0} 2}$ in $P_{0}$.

Let $c_{1}$ be any root of the equation

$$
\phi_{1}(c)=\alpha_{0}^{(1)}+\alpha_{1}^{(1)} c+\cdots+\alpha_{0_{1}}^{(1)} c^{\sigma_{1}}=0 .
$$

We put $w=c_{1} e^{\rho_{1}{ }^{z}}+w^{\prime}$. Then (2) gives an equation for $w^{\prime}$,

$$
f_{1}\left(w^{\prime}\right)=P_{n}^{\prime}(z) w^{\prime n}+\cdots+P_{1}^{\prime}(z) w^{\prime}+P_{0}^{\prime}(z)=0,
$$

where $P_{n}^{\prime}=P_{n}$, and where every $P^{\prime}$ is a function represented by a Dirichlet series with a half-plane of absolute convergence, whose exponents are the $\lambda$ 's plus integral multiples of $\rho_{1}$. The exponents in the $P^{\prime \prime}$ s will have expressions like the second member of (4).

If $P_{0}^{\prime} \equiv 0$, the function $w=c_{1} e^{\rho_{1}{ }^{z}}$ is a solution of (2). If $P_{0}^{\prime} \not \equiv 0$, we treat $f_{1}\left(w^{\prime}\right)$ as we $\operatorname{did} f(w)$, determining a $\rho_{2}$ and a $c_{2}$, then making the substitution $w^{\prime}=c_{2} e^{\rho_{2}}+w^{\prime \prime}$.

We build, in this way, a series

$$
c_{1} e^{\rho_{1} z}+c_{2} e^{\rho_{2} z}+c_{3} e^{\rho_{3} z}+\cdots .
$$

If, at some stage, a $P_{0}{ }^{(j)}$ is found which is identically zero, the series (8) is finite and is a solution of (2), of type (3).

4. We assume, in what follows, that (8) is an infinite series. We say that

$$
\rho_{1}>\rho_{2}>\rho_{3}>\cdots ; \lim _{p \rightarrow \infty} \rho_{p}=-\infty .
$$

(b) The $\rho$ 's are of the form (4).

(c) The series (8) has a half-plane of absolute convergence.

(d) The function defined by (8) satisfies (2).

We remark that $(b)$ is obvious on the basis of $\S 3$.

5. The equation which defines $c_{2}$ in (8) is

$$
\phi_{2}(c)=\alpha_{0}^{(2)}+\cdots+\alpha_{g_{2}}^{(2)} c^{0,}=0 .
$$

We are going to show that if $c_{1}$ is a root of (6) of multiplicity $s_{1}$, then

* In §5, we follow closely Hensel und Landsberg, Algebraische Funktionen, Leipzig, 1902, pp. 53 et seq. In $\$ \S 6,7$, however, situations peculiar to the Dirichlet series problem require us to deviate from the methods used in algebraic function theory. 
$g_{2} \leqq s_{1}$. We prove also that $\rho_{p+1}<\rho_{p}$ for every $p$.

For $c$ arbitrary, we have

$$
f\left(c e^{\rho_{1} z}\right)=\phi_{1}(c) e^{\sigma} 0^{z}+\psi_{1}(c, z) e^{\tau z},
$$

where $\psi_{1}(c, z)$ is a polynomial in $c$ with coefficients which are Dirichlet series with non-positive exponents, and where $\tau<\sigma_{0}$.

If, in (10), we put $c=c_{1}+w^{\prime} e^{-\rho_{1} z}$, the first member becomes $f\left(c_{1} e^{\rho_{1} z}+w^{\prime}\right)$ which is $f_{1}\left(w^{\prime}\right)$. Hence

$$
f_{1}\left(w^{\prime}\right)=\phi_{1}\left(c_{1}+w^{\prime} e^{-\rho_{1} z}\right) e^{\sigma_{0} z}+\psi_{1}\left(c_{1}+w^{\prime} e^{-\rho_{1} z}, z\right) e^{\tau z} .
$$

Comparing this result with (7), we find that

$$
P_{i}^{\prime}=e^{\left(\sigma_{0}-i \rho_{1}\right) z} \frac{\phi_{1}^{(i)}\left(c_{1}\right)}{i !}+e^{\left(r-i \rho_{1}\right) z} \frac{\psi_{1}^{(i)}\left(c_{1}, z\right)}{i !}
$$

where the superscript $(i)$ indicates $i$ differentiations with respect to $c$.

As $\tau<\sigma_{0}$, we find, indicating by accents the $\sigma$ 's for (7), that, if $\phi_{1}{ }^{(i)}\left(c_{1}\right) \neq 0$, then

$$
\sigma_{i}^{\prime}=\sigma_{0}-i \rho_{1}
$$

but that, if $\phi_{1}{ }^{(i)}\left(c_{1}\right)=0$,

$$
\sigma_{i}^{\prime}<\sigma_{0}-i \rho_{1} \text {. }
$$

Thus, if $c_{1}$ is a root of (6) of multiplicity $s_{1}$, we have (14) for $i<s_{1}$, in particular, for $i=0$, and (13) for $i=s_{1}$.

We have

$$
\frac{\sigma_{0}^{\prime}-\sigma_{i}^{\prime}}{i}=\frac{\sigma_{0}^{\prime}-\sigma_{0}}{i}+\frac{\sigma_{0}-\sigma_{i}^{\prime}}{i}
$$

From (13) and (14), we see that $\left(\sigma_{0}-\sigma_{i}^{\prime}\right) / i$ is a minimum, namely $\rho_{1}$, for $i=s_{1}$. As (14) holds for $i=0$, we have $\sigma_{0}^{\prime}<\sigma_{0}$, so that $\left(\sigma_{0}^{\prime}-\sigma_{0}\right) / i$ is greater for $i>s_{1}$ than for $i=s_{1}$. Hence the greatest value of $i$ for which the first member of (15) is a minimum cannot exceed $s_{1}$. This proves that $g_{2} \leqq s_{1}$.

Furthermore, for $i=s_{1}$, the first member of (15) is $\rho_{1}$ decreased by $\left(\sigma_{0}-\sigma_{0}^{\prime}\right) / s_{1}$, so that $\rho_{2}$, the minimum of the first member of (15), is less than $\rho_{1}$. Similarly $\rho_{p+1}<\rho_{p}$ for every $p$.

6. We are going to prove (c) of $\$ 4$, and to complete the proof of (a).

With every positive integer $p$, we have associated a $c_{p}, \rho_{p}, g_{p}$, and $s_{p}$. For every $p$,

$$
g_{p+1} \leqq s_{p} \leqq g_{p} \leqq n
$$


Thus there exists a $q$ such that

$$
g_{p}=s_{p}=g_{q}
$$

for $p \geqq q$. Let $g_{q}$ be represented by $s$. For $p \geqq q$, the $s$ roots of $\phi_{p}(c)=0$ are all equal. Then

$$
\phi_{p}(c)=b_{p}\left(c-c_{p}\right)^{s}
$$

with $b_{p}$ a constant.

We consider the relation, analogous to (10),

$$
f_{p-1}\left(c e^{\rho p z}\right)=\phi_{p}(c) e^{\sigma_{0}^{(p-1)_{z}}}+\psi_{p}(c, z) e^{\tau^{(p-1)_{z}}},
$$

and put in it $c=w^{(p-1)} e^{-\rho} p^{z}$. We find, for $p \geqq q$,

$$
f_{p-1}\left(w^{(p-1)}\right)=b_{p}\left(w^{(p-1)} e^{-\rho_{p} z}-c_{p}\right)^{s} e^{\sigma_{0}^{(p-1)_{z}}}+\psi_{p}\left(w^{(p-1)} e^{-\rho_{p} z}, z\right) e^{\gamma^{(p-1)_{z}}} .
$$

Differentiating $s-1$ times with respect to $w^{(p-1)}$, we find

$$
\begin{aligned}
& f_{p-1}^{(s-1)}\left(w^{(p-1)}\right)=s ! b_{p} e^{\left(\sigma_{0}^{(p-1)}-(s-1) \rho_{p}\right) z}\left(w^{(p-1)} e^{-\rho_{p} z}-c_{p}\right) \\
& \quad+\psi_{p}^{(8-1)}\left(w^{(p-1)} e^{-\rho_{p} z}, z\right) e^{\left.(\tau-1)-(s-1) \rho_{p}\right) z} .
\end{aligned}
$$

The expression $\psi_{p}^{(s-1)}$ in (16) is the result of replacing $c$ by $w^{(p-1)} e^{-p_{p}}$ in the $(s-1)$ th derivative with respect to $c$ of $\psi_{p}(c, z)$.

We shall now find a function $w^{(p-1)}$ of $z$ such that

$$
\underset{p-1}{(s-1)}\left(w^{(p-1)}\right)=0 .
$$

We must have, by (16),

$$
w^{(p-1)}=c_{p} e^{\rho_{p} z}-\frac{1}{s ! b_{p}} \psi_{p}^{(s-1)}\left(w^{(p-1)} e^{-\rho_{p} z}, z\right) e^{\left(\tau(p-1)-\sigma_{0}(p-1)\right) z+\rho_{p} z} .
$$

We put

$$
w^{(p-1)}=\left(u+c_{p}\right) e^{\rho_{p} z}
$$

Then (17) becomes.

$$
u=-\frac{1}{s ! b_{p}} \psi_{p}^{(s-1)}\left(u+c_{p}, z\right) e^{\left(\tau(p-1)-\sigma_{0}(p-1)\right)_{z}} .
$$

Since $\psi_{p}^{\left({ }^{(8-1)}\right.}\left(u+c_{p}, z\right)$ is a polynomial in $u$ whose coefficients are absolutely convergent Dirichlet series with non-positive exponents, and since $\tau^{(p-1)}<\sigma_{0}{ }^{(p-1)}$, we can write (18)

$$
u=Q_{0}+Q_{1} u+\cdots+Q_{n} u^{n},
$$


where the $Q$ 's are absolutely convergent Dirichlet series, with negative exponents, of the type (4). When $x \rightarrow+\infty$, every $Q$ approaches 0 .

Now if the $Q$ 's are regarded as independent variables, (19) has a unique solution for $u$ which is analytic when every $Q$ is small, and which equals 0 when the $Q$ 's are all zero. Let this solution be

$$
u=F\left(Q_{0}, \cdots, Q_{n}\right),
$$

where $F$ is a series of positive powers in the $Q$ 's. If the $Q$ 's are replaced by the Dirichlet series which correspond to them, $u$ becomes a Dirichlet series with a half-plane of absolute convergence,

$$
u=d_{1} e^{\tau_{1} z}+d_{2} e^{\tau_{2} z}+\cdots,
$$

where $0>\tau_{1}>\tau_{2}>\cdots, \lim \tau_{m}=-\infty$.

Then the equation $f_{p-1}^{(s-1)}\left(w^{(p-1)}\right)=0$ is satisfied by the Dirichlet series

$$
w^{(p-1)}=c_{p} e^{\rho_{p} z}+d_{1} e^{\left(\tau_{1}+\rho_{p}\right) z}+\cdots,
$$

which has a half-plane of absolute convergence.

Now

$$
f_{p-1}^{(s-1)}\left(w^{(p-1)}\right)=f^{(s-1)}\left(c_{1} e^{\rho_{1} z}+\cdots+c_{p-1} e^{\rho_{p-1} z}+w^{(p-1)}\right) .
$$

Hence the equation

$$
f^{(s-1)}(w)=0
$$

is satisfied by

$$
w=c_{1} e^{\rho_{1} z}+\cdots+c_{p} e^{\rho_{p} z}+d_{1} e^{\left(\tau_{1}+\rho_{p}\right) z}+\cdots \cdot
$$

For every $p$, there is a solution of (22) of type (23). Of course, the $\tau$ 's and $d$ 's, in (21), depended, as far as we knew, on $p$, but as (22) has at most $n$ solutions, and as two distinct series (23) cannot represent the same function, there can be at most $n$ series (23) for the various values of $p$. One of these series must be the series (8). Hence $\lim \rho_{p}=-\infty$, and (8) has a half-plane of absolute convergence. This proves (a) and (c).

7. To prove (d), it will suffice to show that (8) satisfies (2) formally. This will follow if we can show that $\sigma_{0}^{(p)} \rightarrow-\infty$ as $p \rightarrow \infty$. As $f_{p}$ is obtained from $f$ by replacing $w$ by $w^{(p)}$ plus a segment of (8), it is obvious that an $M$ exists such that $\sigma_{i}^{(p)}<M$ for every $i$ and $p$. Now, for $p \geqq q$, by (13),

$$
M>\sigma_{s}{ }^{(p+1)}=\sigma_{0}^{(p)}-s \rho_{p+1}
$$

so that $\sigma_{0}^{(p)}<M+s \rho_{p+1}$, and $\sigma_{0}^{(p)} \rightarrow-\infty$, because $\rho_{p+1} \rightarrow-\infty$. 


\section{Dirichlet SERIES With COMPLEX EXPonents}

8. In what follows, series represented by small letters (as $a(z))$ will be series

$$
v_{1} e^{\mu_{1} i z}+\cdots+v_{p} e^{\mu_{p} i z}+\cdots,
$$

where the $\mu$ 's are real and either increase with their subscripts, tending toward $+\infty$, or else decrease as their subscripts increase, tending toward $-\infty$. The $v$ 's are constants.

Series represented by large letters (as $P(z)$ ) will be of the form

$$
a_{1}(z) e^{\lambda_{1} z}+\cdots+a_{p}(z) e^{\lambda_{p} z}+\cdots,
$$

where the $\lambda$ 's are real and decreasing, with $-\infty$ as their limit. The $a$ 's are of type (24), and while the $\mu$ 's in any two of them need not be the same, the $\mu$ 's must either increase with their subscripts in all $a$ 's, or else decrease in all $a$ 's.

9. We shall use, in what follows, sectors of the complex plane bounded by two half-lines which emanate from a point. The boundary of a sector will be understood to belong to the sector.

10. If $a(z)$ is of type (24), we shall use $\bar{a}(z)$ to represent the sum of the moduli of the terms of $a(z)$.

We shall say that (25) converges normally in a sector, if every $\bar{a}(z)$, and also

$$
\sum_{p=1}^{\infty} \bar{a}_{p}(z)\left|e^{\lambda p z}\right|
$$

converges in the sector, and if, for every $h>0$, a positive integer $q$ exists such that

$$
\sum_{p=q+1}^{\infty} \bar{a}_{p}(z)\left|e^{\lambda p z}\right|<e^{-h|z|}
$$

for $z$ in the sector.

11. We prove that if $P(z)$ is normally convergent in a sector $S$, then $P(z)$ is uniformly convergent in $S$.

It suffices to prove that $P(z)$ is uniformly convergent in any finite triangular part of $S$. The normal convergence of $P(z)$ will then guarantee that the convergence is uniform throughout $S$.

The uniformity of the convergence in any triangle is essentially a result of Hille. ${ }^{*}$ Let the vertices of the triangle be $z_{1}, z_{2}, z_{3}$. Then any $z$ within or on the boundary of the triangle has a representation

\footnotetext{
* Hille, p. 263.
} 


$$
z=\gamma_{1} z_{1}+\gamma_{2} z_{2}+\gamma_{3} z_{3},
$$

where the $\gamma$ 's are non-negative numbers whose sum is unity. Then, for any $\beta$,

$$
\left|e^{\beta z}\right|=\left|\left(e^{\beta z_{1}}\right)^{\gamma_{1}}\left(e^{\beta z_{2}}\right) \gamma_{2}\left(e^{\beta z_{3}}\right) \gamma_{3}\right| \leqq \max \left|e^{\beta z_{i}}\right|, \quad i=1,2,3 .
$$

It is thus clear that the remainder of (25) at any point in the triangle has a modulus not greater than the sum of the corresponding remainders of (26) at the points $z_{1}, z_{2}, z_{3}$. This shows that the convergence is uniform.

If (26) is convergent in $S$, we know that $P(z)$ converges uniformly in any finite part of $S$. Thus to prove that $P(z)$ is normally convergent in $S$, it suffices to show that (26) converges in $S$ and that for every $h>0$, an $r>0$ can be found such that some remainder of (26) is less than $e^{-h|z|}$ for $|z|>r$.

12. Let $S$ be a sector with one side extending in the direction of the positive real axis. Let

$$
P(z)=\sum a_{p}(z) e^{\lambda_{p} z}, \quad Q(z)=\sum b_{p}(z) e^{\lambda_{p} z},
$$

where each pair $a_{p}$ and $b_{p}$ have the same $\mu$ 's, be normally convergent in $S$. Furthermore, suppose that $P(z)=Q(z)$ in $S$. We shall prove that $a_{p}(z) \equiv b_{p}(z)$ for every $p$.

It will suffice to prove that $a_{1}(z) \equiv b_{1}(z)$.

Consider any half-line in $S$ which is directed like the positive real axis. We have, in $S$,

$$
a_{1}(z)+\sum_{p=2}^{\infty} a_{p}(z) e^{\left(\lambda_{p}-\lambda_{1}\right) z}=b_{1}(z)+\sum_{p=2}^{\infty} b_{p}(z) e^{\left(\lambda_{p}-\lambda_{1}\right) z} .
$$

Because the two series in (28) are normally convergent, the remainder after a large number of terms in either series approaches zero as $z$ goes to $\infty$ on the half-line taken above. But as every $\lambda_{p}-\lambda_{1}$ is negative for $p>1$ and as every $a_{p}$ and $b_{p}$ is bounded on the half-line, the sum of any finite number of terms of either series approaches zero as $z$ increases on the half-line. Hence, for such an increase of $z$, both series approach 0 . Now $a_{1}(z)$ and $b_{1}(z)$, functions defined by absolutely convergent Dirichlet series, are almost periodic, with real quasi-periods. Thus there is a sequence of positive numbers $h_{1}, \ldots$, $h_{p}, \cdots$, tending toward $+\infty$, such that, for any $z$ on the half-line,

$$
a_{1}\left(z+h_{p}\right) \rightarrow a_{1}(z), \quad b_{1}\left(z+h_{p}\right) \rightarrow b_{1}(z)
$$

as $p \rightarrow \infty$.* This, by (28), means that $a_{1}(z)=b_{1}(z)$ for every $z$ on the half-line. Hence $a_{1}(z)$ is identical with $b_{1}(z)$.

\footnotetext{
* Bohr, Acta Mathematica, vol. 45 (1925), p. 37.
} 
13. We consider two series of type (25)

$$
P=\sum a_{p}(z) e^{\lambda p z}, \quad Q=\sum b_{p}(z) e^{\lambda p z}
$$

where $a_{p}$ and $b_{p}$ have the same $\mu$ 's, which tend toward $+\infty$. We assume that $P$ and $Q$ are both normally convergent in a sector $S$, which has one side extending in the direction of the positive real axis, and whose second side is inclined at a positive angle less than $\pi$ to the first one. It is understood that $S$ is generated by a positive rotation from its first side to its second.*

The product $P Q$ can be written as a series of type (25), $\sum c_{p}(z) e^{-\lambda_{p}{ }^{\prime z}}$ where each $\lambda^{\prime}$ is a sum of two $\lambda$ 's and where each $c$ is a bilinear combination of $a$ 's and $b$ 's. Furthermore, every $\bar{c}_{p}(z)$ and also $\sum \bar{c}_{p}(z)\left|e^{\lambda} p^{\prime z}\right|$ will be convergent in $S$.

We are going to prove that $P Q$ is normally convergent in $S$.

It is easy to see that the product of $P$ by an $e^{\lambda z}$ or by an absolutely convergent series of type (24), with increasing $\mu$ 's, is normally convergent in $S$. (Cf. final remarks of $\$ 11$.) Hence the product of $P$ by the sum of a finite number of terms of $Q$ is normally convergent in $S$.

Let $h>0$ be assigned arbitrarily, and let $q$ be taken so that

$$
\sum_{p=q+1}^{\infty} \bar{a}_{p}(z)\left|e^{\lambda p z}\right|<e^{-h|z|}, \quad \sum_{p=q+1}^{\infty} \bar{b}_{p}(z)\left|e^{\lambda p z}\right|<e^{-h|z|}
$$

in $S$. Let, furthermore,

$$
\begin{aligned}
& P=a_{1}(z) e^{\lambda_{1} z}+\cdots+a_{q}(z) e^{\lambda_{q} z}+P_{q}(z), \\
& Q=b_{1}(z) e^{\lambda_{1} z}+\cdots+b_{q}(z) e^{\lambda_{q}}+Q_{q}(z) .
\end{aligned}
$$

Now $P Q$ is the sum of an infinite number of infinite series of the form $c(z) e^{\lambda^{\prime} z}$. We shall consider $P Q$ as a double series of terms $v e^{\left(\mu i+\lambda^{\prime}\right) z}$. Those terms of the double series which come from multiplying terms of $P_{q}$ and $Q_{q}$ have less than $e^{-2 h|z|}$ for the sum of their moduli. From this, and from the fact that the product of $P_{q},\left(Q_{q}\right)$, by the sum of the first $q$ terms of $Q,(P)$, is normally convergent in $S$, it follows that $P Q$ is normally convergent in $S$.

Of course, we can derive a similar result for $a$ 's and $b$ 's with decreasing $\mu$ 's, provided that the second side of $S$ makes a negative angle less than $\pi$ with the first side.

14. Let $S$ be a sector which has one side extending in the direction of the positive real axis, and whose second side makes a positive angle less than $\pi$ with the first one. $\dagger$ (See Fig. 1.)

* This will always be understood when we say that the opening of a sector is a positive angle.

$\dagger$ The assumption that the opening is less than $\pi$ has no bearing on our final results, but it contributes simplicity to our discussion. 
Let $Q_{1}, \cdots, Q_{n}$ be $n$ series of type (25), with negative $\lambda$ 's and increasing $\mu$ 's, all normally convergent in $S$.

Let $u=F\left(u_{1}, \cdots, u_{n}\right)$ be a function analytic in all of its arguments at $(0, \cdots, 0)$.

Let $\bar{Q}_{i}$ be the series (26) for $Q_{i}$. Consider any term $\bar{a}(z)\left|e^{\lambda_{z}}\right|$ of $\bar{Q}_{i}$. Because $\lambda<0$, there is an $h>0$ such that $\bar{a}(z)\left|e^{\lambda z}\right|<e^{-h|z|}$ for $z$ sufficiently large, in a sector whose first side is the horizontal side of $S$ and whose second side makes a sufficiently small positive angle with the first one. For, if the opening of the new sector is made sufficiently small, $\bar{a}(z)$, if it becomes infinite at all, as $z \rightarrow \infty$, will become infinite like $e^{k|z|}$ with $k$ small.

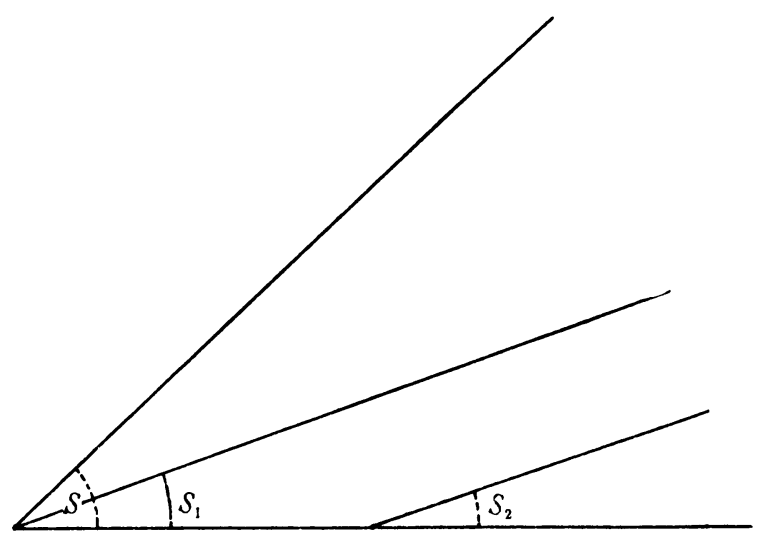

Fig. 1

From this fact, and from the normal convergence of $Q_{i}$, we see that there is an $h>0$ and a sector $S_{1}$, contained in $S$ and with one side coincident with the horizontal side of $S$, such that, for every $i, \bar{Q}_{i}<e^{-h|z|}$ for $z$ large and in $S_{1}$. In what follows, we suppose an $h>0$ and an $S_{1}$ to have been found, and we hold them fixed.

Let $\bar{F}$, a majorant for $F\left(u_{1}, \cdots, u_{n}\right)$, converge for $\left|u_{i}\right|<\delta$, where $\delta>0(i=1, \cdots, n)$. Let $S_{2}$ be a sector contained in $S_{1}$, with vertex on the horizontal side of $S_{1}$, with one side horizontal and with its second side parallel to the second side of $S_{1}$, such that $\bar{Q}_{i}<\delta$ in $S_{2}$, for every $i$.

When each $u_{i}$ is replaced by $Q_{i}$ in $u=F\left(u_{1}, \cdots, u_{n}\right), u$ will become a series $U(z)$ of type (25), absolutely convergent (considered as a double series) in $S_{2}$.

We say that $U(z)$ is normally convergent in $S_{2}$ s

For $t$ any integer, arbitrarily large, let

$$
F\left(u_{1}, \cdots, u_{n}\right)=A\left(u_{1}, \cdots, u_{n}\right)+B\left(u_{1}, \cdots, u_{n}\right),
$$


where $A$ consists of those terms of $F$ which are of degree not exceeding $t$, and where $B$ consists of the remaining terms of $F$. Let $\bar{B}$ be the majorant of $B$. Then there is an $\eta>0$, depending on $t$, such that, for $\left|u_{i}\right|<\eta$,

$$
|\bar{B}| \leqq \max \left(\left|u_{1}\right|^{t}, \cdots,\left|u_{n}\right|^{t}\right) \text {. }
$$

When each $u_{i}$ is replaced in $A$ by $Q_{i}$, we get, according to $\S 13$, a series (25) normally convergent in $S$, hence in $S_{2}$. But, by (29), $B$ will yield a double series the sum of the moduli of the terms of which is less than $e^{-h t|z|}$ for $z$ large in $S_{1}$. Furthermore, $t$ may be taken arbitrarily large. These facts and the final remarks of $\$ 11$, show that $U(z)$ converges normally in any sector contained in $S_{1}$ in which $U(z)$ converges (as a double series) absolutely. The sector $S_{2}$ is such a sector contained in $S_{1}$.

A similar result holds for the case where the $\mu$ 's are decreasing and the second side of $S$ makes a negative angle with the first.

15. We consider a sector $A M B$ (Fig. 2) consisting of those points $z$, for which

$$
|\operatorname{amp}(z-M)| \leqq \theta,
$$

where $0<\theta<\pi$, the point $M$ being on the real axis. Let $O_{1}$ be on $M A, O_{2}$ be conjugate to $O_{1}$. Let $O_{1} C_{1}$ and $O_{2} C_{2}$ be horizontal half-lines having the direction of the positive real axis.

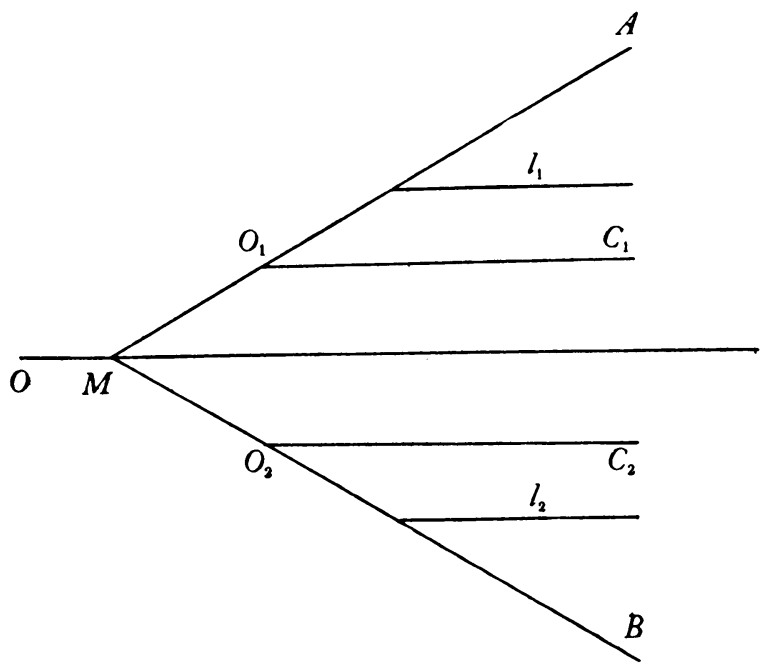

FIg. 2

Let $\phi(z)$ be a function analytic in the sector $A M B$. Let $\phi(z)$ have in $A O_{1} C_{1}$ a normally convergent representation

$$
a_{1}(z) e^{\lambda_{1} z}+\cdots+a_{p}(z) e^{\lambda_{p} z}+\cdots
$$


with increasing $\mu$ 's, and, in $\mathrm{BO}_{2} \mathrm{C}_{2}$, a normally convergent representation

$$
b_{1}(z) e^{\lambda_{1} z}+\cdots+b_{p}(z) e^{\lambda_{p} z}+\cdots
$$

with the same* $\lambda$ 's which appear in (30) but with decreasing $\mu$ 's.

Furthermore suppose that a $k>0$ exists such that $|\phi(z)|<e^{k|z|}$ in $A M B$.

We say that

(a) For every $p, a_{p}(z)$ and $b_{p}(z)$ are exponential polynomials, and $a_{p}(z) \equiv b_{p}(z)$.

(b) The identical series (30) and (31) converge normally to $\phi(z)$ in a sector interior to $A M B$, with vertex on the real axis, and with sides parallel to those of $A M B$.

In proving (a), we may limit ourselves to the case of $p=1$.

Let $l_{1}$ and $l_{2}$ be horizontal half-lines respectively interior to $A O_{1} C_{1}$ and $\mathrm{BO}_{2} \mathrm{C}_{2}$ and at equal distances from the real axis. We take any half-strip between $l_{1}$ and $l_{2}$, that is, a region contained in $A M B$, limited by $l_{1}, l_{2}$ and a segment perpendicular to $l_{1}$ and $l_{2}$.

Consider the function $\psi(z) e^{-\lambda_{1} z} \phi(z)$. By (30), (31), it is bounded on $l_{1}$ and $l_{2}$. Hence as $|\psi(z)|<e^{\left(k+\left|\lambda_{1}\right|\right)|z|}$, in the half-strip between $l_{1}$ and $l_{2}, \psi(z)$, according to the Phragmen-Lindelöf theorem, must be bounded in the halfstrip.

The functions $a_{1}(z)$ and $b_{1}(z)$ are almost periodic, with real quasi-periods, on $O_{1} C_{1}$ and $O_{2} C_{2}$ respectively. We consider a sequence of positive numbers $h_{1}, \cdots, h_{p}, \cdots$, increasing to infinity, such that for $z$ on $O_{1} C_{1}, a_{1}\left(z+h_{p}\right)$ $\rightarrow a_{1}(z)$ as $p \rightarrow \infty$, and such that, for $z$ on $O_{2} C_{2}, b_{1}\left(z+h_{p}\right) \rightarrow b_{1}(z)$ as $p \rightarrow \infty$.

Then $\psi\left(z+h_{p}\right) \rightarrow a_{1}(z)$ on $O_{1} C_{1}$ and $\psi\left(z+h_{p}\right) \rightarrow b_{1}(z)$ on $O_{2} C_{2}$ as $p \rightarrow \infty$.

Consider any rectangle cut from the half-strip between $l_{1}$ and $l_{2}$ by vertical lines. In such a rectangle, the functions $\psi\left(z+h_{p}\right)$ have a common upper bound for their moduli. The sequence $\psi\left(z+h_{p}\right)$ converges on two horizontal lines inside of the rectangle. By the Vitali-Porter theorem, the sequence converges uniformly in any area interior to the rectangle. The analytic limit approached by $\psi\left(z+h_{p}\right)$ equals $a_{1}(z)$ on $O_{1} C_{1}$ and $b_{1}(z)$ on $O_{2} C_{2}$. Hence $a_{1}(z)$ and $b_{1}(z)$ are continuations of each other. That is, there is a function $c(z)$, analytic in a right half-plane $T$, which coincides with $a_{1}(z)$ above $O_{1} C_{1}$, and with $b_{1}(z)$ below $\mathrm{O}_{2} \mathrm{C}_{2}$. Furthermore, $c(z)$ is bounded in any horizontal half-strip of $T$.

It is now easy to see that $a_{1}(z)$ contains only a finite number of $\mu$ 's. For if $v_{m} e^{\mu} m^{i z}$ is a term of $a_{1}(z)$, then, $\omega$ being a positive variable,

$$
v_{m}=\lim _{\omega \rightarrow \infty} \frac{1}{\omega} \int_{O_{1}}^{O_{1}+\omega} e^{-\mu_{m} i z} c(z) d z
$$

\footnotetext{
* The assumption that the $\lambda$ 's are the same is not a restrictive on
} 
the integration being performed along $O_{1} C_{1}$. Now, as $e^{-\mu_{m}{ }^{i z}} c(z)$ is bounded in any horizontal half-strip of $T$, we have also

$$
v_{m}=\lim _{\omega \rightarrow \infty} \frac{1}{\omega} \int_{O_{2}}^{O_{2}+\omega} e^{-\mu_{m} i z} c(z) d z .
$$

For the difference between the integrals in (32) and (33) is

$$
\int_{o_{1}}^{o_{2}}-\int_{0_{1}+\omega}^{o_{2}+\omega}
$$

which is bounded for all $\omega$ 's.

Imagine that $\mu_{m}$ exceeds the first $\mu$ in $b_{1}(z)$. As the $\mu$ 's in $b_{1}(z)$ decrease, $\mu_{m}$ will be distinct from every $\mu$ in $b_{1}(z)$. By (33) we will have $v_{m}=0$. Hence $a_{1}(z)$ has only a finite number of $\mu$ 's and is an exponential polynomial. The series $b_{1}(z)$ is an exponential polynomial identical with $a_{1}(z)$.

To prove (b), we consider any term $w(z)=v e^{(\mu i+\lambda) z}$ of (30) regarded as a double series. Let $z$ be any point between $O_{1} C_{1}$ and $O_{2} C_{2}$, and $z_{1}, z_{2}$ two points of the same abscissa as $z$, but respectively above $O_{1} C_{1}$ and below $O_{2} C_{2}$. Then*

where $0<k<1$. Hence

$$
z=(1-k) z_{1}+k z_{2}
$$

$$
|w(z)|=\left|w\left(z_{1}\right)\right|^{1-k}\left|w\left(z_{2}\right)\right|^{k} \leqq \max \left(\left|w\left(z_{1}\right)\right|,\left|w\left(z_{2}\right)\right|\right),
$$

and this is easily seen to imply (b).

16. Let

$$
P(z)=\sum a_{p}(z) e^{\lambda_{p} z}, \quad Q(z)=\sum b_{p}(z) e^{\lambda p z},
$$

with every $a(z)$ and every $b(z)$ an exponential polynomial, be normally convergent in a sector $S$, of opening less than $2 \pi$, which is symmetrical with respect to, and contains an infinite portion of, the positive real axis.

Suppose that $P / Q$ is analytic in $S$.

We shall prove that there is a sector $T$, contained in $S$, symmetric with respect to, and containing an infinite portion of, the positive real axis, in which $P / Q$ admits a normally convergent expansion $\sum c_{p}(z) e^{\lambda^{\prime} z}$, with every $c(z)$ an exponential polynomial.

We shall show first that in some sector $S_{1}$, contained in $S$, with one side in the direction of the positive real axis, and generated by a positive rotation less than $\pi$, the quotient $P / Q$ has a normally convergent expansion (25) with increasing $\mu$ 's.

Let $t$ be the smallest value of $p$ for which $b_{p}(z) \not \equiv 0$. Then $Q(z)=b_{t}(z) e^{\lambda} t^{z}$

* Cf. Hille, p. 263. 
$(1+R(z))$, where $R(z)$ has a normally convergent expansion (25), with increasing $\mu$ 's, and with negative $\lambda$ 's, in a sector $U$ like the $S_{1}$ just described. By $\$ 14,1 /(1+R(z))$ has a normally convergent expansion (25) in a sector $V$ with one side horizontal, interior to $U$. Then $1 / Q(z)$ has a normally convergent expansion (25) in a sector $S_{1}$, described as above, interior to $V$. By $\$ 13, P / Q$ has an expansion (25) with increasing $\mu$ 's, normally convergent in $S_{1}$.

Similarly, in a sector $S_{2}$, generated by a negative rotation, $P / Q$ has a normally convergent expansion (25) with decreasing $\mu$ 's.

We are going to show that the expansions in $S_{1}$ and $S_{2}$ are identical, and that the $a(z)$ 's in them are exponential polynomials. This, according to the proof of (b) of $\$ 15$, will give us our result.

Let $a_{q}(z)$ and $b_{t}(z)$ be, respectively, the first $a(z)$ and $b(z)$ which are not identically zero. We shall show that $a_{q}(z) / b_{t}(z)$ is an exponential polynomial with pure imaginary exponents.

It is no specialization to assume that $q=t=1$ and that $\lambda_{1}=0$. These circumstances may be brought about by multiplying $P$ and $Q$ by exponentials and using a new set of $\lambda$ 's.

There exists a horizontal strip $L$ which contains all of the zeros of $b_{1}(z)$ in its interior. Let $B$ be the difference between the greatest and least $\mu$ 's in $b_{1}(z)$. The number of zeros of $b_{1}(z)$ whose abscissas lie between $u$ and $v(u<v)$ is equal to*

$$
\frac{B(v-u)}{2 \pi}+O(1)
$$

We may obviously take $L$ so broad that $b_{1}(z)$ is bounded away from zero along the sides of $L$. We suppose this to be done.

Because $b_{1}(z)$ is almost periodic, we can break $L$ up into an infinite number of rectangles, whose lengths have a common upper bound, such that for some $h>0,\left|b_{1}(z)\right|>h$ along all of the vertical sides of the rectangles.

Now $Q(z)=b_{1}(z)+R(z)$, where $R(z)$ has negative $\lambda$ 's. $R(z)$ approaches zero as $z$ goes towards $\infty$ in $L$, in the right-hand direction.

Consequently, in any of the rectangles mentioned above, situated at a great distance to the right, $Q(z)$ has the same number of zeros as $b_{1}(z)$. It follows easily that the number of zeros of $Q(z)$, in the region common to $L$ and to $S$, with abscissas between $u$ and $v$, is equal to

$$
\frac{B(v-u)}{2 \pi}+O(1) \text {. }
$$

* Tamarkin, p. 24, P6́lya, Schwengler, Wilder, p. 420, Ritt (1). 
Suppose that $a_{1} / b_{1}$ is not an exponential polynomial. Then it is possible to find an exponential polynomial $g(z)$, with pure imaginary exponents, the difference between whose greatest and least $\mu$ 's is less than $B$, above, such that

$$
a_{1}(z)=d(z) b_{1}(z)+g(z),
$$

with $d(z)$ an exponential polynomial having pure imaginary exponents.*

We consider the function $P(z)-d(z) Q(z)$, which, because $P$ vanishes wherever $Q$ does, in $S$, also vanishes where $Q$ does, in $S$.$\} We have$

$$
P(z)-d(z) Q(z)=g(z)+K(z),
$$

where $K(z)$ is a series of type (25), with negative $\lambda$ 's. This means, by the discussion leading up to (35), that $P(z)-d(z) Q(z)$ has fewer zeros than $Q(z)$ in a long horizontal rectangle. We have here a contradiction which shows that $a_{1}(z) / b_{1}(z)$ is an exponential polynomial.

Thus $a_{q}(z) / b_{t}(z)$ is, as we stated, an exponential polynomial, $c_{1}(z)$.

Now the first coefficient in the expansion of $1 / Q$ in $S_{1}$ is $1 / b_{t}(z)$ expanded with increasing $\mu$ 's. Hence the first coefficient in the expansion (25) of $P / Q$ is $a_{q}(z) / b_{t}(z)$, which is $c_{1}(z)$. The same is true of the expansion of $P / Q$ in $S_{2}$.

Let the first term in the expansion of $P / Q$, either in $S_{1}$ or in $S_{2}$, be $c_{1}(z) e^{\lambda_{1}{ }^{\prime} z}$.

The second term in the expansion of $P / Q$, say in $S_{1}$, is the first term in the expansion of

$$
\frac{P-c_{1}(z) e^{\lambda_{1}{ }^{\prime} z} Q}{Q} .
$$

Since (36) is analytic in $S$, we know that the first coefficient in its expansion is an exponential polynomial.

Continuing in this way, we find that the developments of $P / Q$ in $S_{1}$ and in $S_{2}$ are identical, and that the coefficients in them are exponential polynomials. We thus have the result stated at the beginning of this article.

17. Let $O X$ (Fig. 3) be the positive real axis, and $O A$ a ray of positive inclination less than $\pi$. Let $S$ be any sector contained in $X O A$, with sides parallel to those of $X O A$. Let

$$
P(z)=a_{1}(z) e^{\lambda_{1} z}+\cdots+a_{p}(z) e^{\lambda_{p} z}+\cdots,
$$

with increasing $\mu$ 's, be normally convergent in $S$.

* Ritt, (1). 
Let $\phi$ be a positive angle less than angle $X O A$. Suppose that, in (37), we replace $z$ by $e^{i \phi} z$.

Consider any term

$$
v e^{\mu i z} e^{\lambda z}
$$

of (37) regarded as a double series. This term goes over into

$$
v e^{(\mu \cos \phi+\lambda \sin \phi) i z} e^{(\lambda \cos \phi-\mu \sin \phi) z} .
$$

We shall prove that, given any real $h$, there are only a finite number of pairs $\mu, \lambda$ such that $\lambda \cos \phi-\mu \sin \phi>h$.

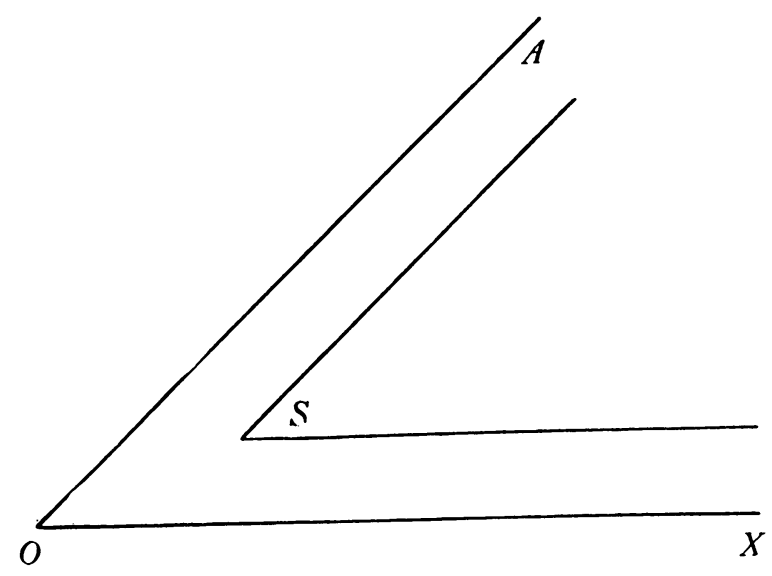

FIG. 3

We shall thus know that $P\left(e^{i \phi} z\right)$ can be written in the form

$$
b_{1}(z) e^{\lambda_{1}^{\prime} z}+\cdots+b_{p}(z) e^{\lambda_{p}^{\prime}}+\cdots,
$$

with $\lambda$ "s decreasing toward $-\infty$, and with every $b$ an exponential polynomial. Furthermore, (40) will converge absolutely to $P\left(e^{i \phi_{z}}\right)$ in the sector obtained by rotating $S$ about the origin through an angle $-\phi$.

For the proof, consider any term of (37). There can be in it only a finite number of terms like (38) for which $\lambda \cos \phi-\mu \sin \phi>h$, for any $h$, since $\sin \phi>0$ and the $\mu$ 's in any term of (37) increase to $+\infty$. Again, because of the normal convergence of (37), a term (38) coming from a large $p$ of (37) must approach zero along the line amp $z=\phi$ like an $e^{-k z}$ with $k$ very large and positive. A similar fact is therefore true of (39) along the positive real axis. Hence $\lambda \cos \phi-\mu \sin \phi$ must be large and negative when $p$ is large. This proves our statement.

Let $T$ be the sector into which $S$ goes, after a rotation about the origin through an angle $-\phi$. Let $U$ be a sector whose vertex is that of $T$, whose 
first side has the inclination $-\phi+\epsilon$, where $\epsilon$ is arbitrarily small and positive, and whose second side is the second side of $T$. That is, $U$ is found from $T$ by cutting away a small sector at the lower edge of $T$.

We say that, in $U,(40)$ converges normally to $P\left(e^{i \phi_{z}}\right)$.

We take any $a_{p}(z)$ of (37), and consider it as a Dirichlet series. For any sector of opening less than $\pi$, symmetrical with respect to the positive imaginary axis, and for any $h>0$, the remainder after a large number of terms in $\vec{a}_{p}(z)$ is less than $e^{-h|z|}$.

Hence, in the sector $U$, the sum of the moduli of those terms (39) which come from a particular $a_{p}(z) e^{\lambda p^{2}}$, and whose $\mu$ 's are sufficiently large, will be less than $e^{-h|z|}$, where $h$ is large at pleasure.

On the other hand, because of the normal convergence of (37) in $S$, we can fix an $m$ such that the terms (39) which come from the remainder after $m$ terms in (37) have, in $T$, a sum for their moduli less than $e^{-h|z|}$.

For $p$ sufficiently great, in (40), the terms (39) in $b_{p}(z) e^{\lambda p^{\prime}}$ come either from a term beyond the $m$ th in (37) or else from terms (38) of large $\mu$ in one of the first $m$ terms of (37).

Thus, for $p$ large,

$$
\sum_{i=p+1}^{\infty} \bar{b}_{i}(z)\left|e^{\lambda_{i}^{\prime} z}\right|<(m+1) e^{-h|z|} .
$$

For any $k<h$, the second member of $(41)$ is, for $|z|$ large, less than $e^{-k|z|}$. This, by the final remarks of $\$ 11$, proves our statement.

\section{EQUations whose COEFFICIENTS have COMPLEX EXPONENTS}

18. We consider an equation

$$
f(w)=P_{n}(z) w^{n}+\cdots+P_{1}(z) w+P_{0}(z)=0,
$$

where every $P(z)$ is of type (25), with increasing $\mu$ 's, normally convergent in a sector $S$ whose first side has the direction of the positive real axis, the sector being generated by a positive rotation less than $\pi$, starting from the first side. We assume that $P_{n}(z) \not \equiv 0$.

We shall prove that the $n$ solutions of (42) are all analytic in a sector $U$ interior to $S$, the sector $U$ having one side in the direction of the positive real axis and being generated by a positive rotation starting from its first side. In $U$, the $n$ solutions will each have a normally convergent representation (25), with increasing $\mu$ 's. Each $\lambda$ in the representations of the solutions will be given by an expression like the second member of (4), in terms of the $\lambda$ 's in the $P$ 's. Each $\mu$ in the representations of the solutions will be similarly expressed in terms of the $\mu$ 's in the $P$ 's. 
For the case in which the $P$ 's have decreasing $\mu$ 's and are normally convergent in a sector generated by a negative rotation, similar results hold, the solutions having representations (25) with decreasing $\mu$ 's.

We assume that $P_{0}(z) \not \equiv 0$. For every $i$, we define $\sigma_{i}$ as in $\S 3$, using series $a(z)$ instead of constants $a$. Similarly, we define $g_{1}$ and $\rho_{1}$.

We consider the equation (6), where now each $\alpha^{(1)}$ is a series (24) with increasing $\mu$ 's. The $g_{1}$ solutions of (6) will be analytic in some upper halfplane, and will have absolutely convergent expansions (24) with $\mu$ 's found from the $\mu^{\prime}$ 's in the $\alpha^{(1)}$ 's by expressions like the second member of (4), with $r$ fixed. Let $c_{1}$ be any solution of (6).

We put, in (42), $w=c_{1} e^{\rho_{1} z}+w^{\prime}$. Then $w^{\prime}$ is determined by (7), where, now, each $P^{\prime}$ is of type (25), normally convergent in $S_{1}$, the sector common to $S$ and the half-plane of absolute convergence of $c_{1}$.

As in $\S 3$, we build a series

$$
c_{1}(z) e^{\rho_{1} z}+c_{2}(z) e^{\rho_{2} z}+\cdots+c_{p}(z) e^{\rho^{p z}}+\cdots .
$$

We continue as in $\S 5$. In $(10), \psi_{1}(c, z)$ is a polynomial in $c$, with coefficients of type (25), which have non-positive $\lambda$ 's and are normally convergent in $S$. In (12), $\psi_{1}{ }^{(i)}\left(c_{1}, z\right)$ is normally convergent in $S_{1}$ and has non-positive $\lambda$ 's. If $s_{1}$ is defined as in $\S 5$, we find again that $g_{2} \leqq s_{1}$. Also, it is seen that $\rho_{2}<\rho_{1}$.

We arrive finally at (19) of $\$ 6$, where now each $Q$ is of type (25), with negative $\lambda$ 's, normally convergent in a sector $S_{p}$ which has one side in the direction of the positive real axis, and whose second side is the second side of $S$.

Let $\bar{Q}_{i}$ be defined as in $\S 14$. We see, as in $\S 14$, that in some sector $T$ whose first side is the horizontal side of $S_{p}$ and whose second side makes a sufficiently small positive angle with the first side, every $\bar{Q}_{i} \rightarrow 0$ as $|z| \rightarrow \infty$.

Hence we can replace every $Q_{i}$ in (20) by its expansion (25), and get an expression (25) for $u$, normally convergent in a sector $U$ with vertex on the horizontal side of $T$, the first side of $U$ being horizontal, and the second side of $U$ being parallel to the second side of $T$.

The function $u$ thus obtained is a solution of (18). It leads, as in $\S \S 6,7$, to a solution of (42) of type (25).

\section{Applications to algebraic combinations of exponentials}

19. We consider now an equation

$$
P_{n} w^{n}+P_{n-1} w^{n-1}+\cdots+P_{0}=0
$$

with every $P$ an exponential polynomial and $P_{n} \not \equiv 0$.

Evidently a function $w$, defined by (43), has, in the finite part of the 
plane, only isolated singularities, which are poles or branch points. When $P_{n} \equiv 1$, there are no poles.

If a sector $T$ is contained in a sector $S$, and if the sides of $T$ have the same directions as those of $S$, we shall call $T$ a subsector of $S$. The vertex of $T$ may be on the boundary of $S$.

Consider a series

$$
v_{1} e^{\sigma_{1} z}+\cdots+v_{p} e^{\sigma_{p} z}+\cdots
$$

with constant $v^{\prime}$ 's, and with real or complex $\sigma$ 's such that, as $p \rightarrow \infty,\left|\sigma_{p}\right| \rightarrow \infty$.

We shall say that (44) is normally convergent in a sector, if it is absolutely convergent in the sector, and if, for every $h>0$, a $q$ exists such that

$$
\sum_{p=q+1}^{\infty}\left|v_{p} e^{\sigma_{p z}}\right|<e^{-h|z|}
$$

throughout the sector. We see, as in $\$ 11$, that (44) converges uniformly in any sector in which it converges normally.

Let $S$ be a sector of opening less than $2 \pi$, and suppose that some branch $w=f(z)$ of a function defined by (43) is analytic in a sector which has the same vertex as $S$, and which contains in its interior every point of $S$ other than the vertex.

We are going to show that, in some subsector of $S, w=f(z)$ has a normally convergent development of type (44). The real part of each $\sigma$ is expressed in terms of the real parts of the exponents of the $P$ 's by a relation like (4), and the imaginary parts of the $\sigma$ 's are expressed analogously.

Let the angles between the sides of $S$ and the positive real axis be $\phi_{1}$ and $\phi_{2}$, where

$$
0 \leqq \phi_{1}<\phi_{2} \leqq 2 \pi, \quad \phi_{2}-\phi_{1}<2 \pi .
$$

Let $\psi$ be such that $\phi_{1} \leqq \psi \leqq \phi_{2}$.

We shall show that $f\left(e^{i} \psi z\right)$ has an expansion (25), with $a$ 's which are exponential polynomials, normally convergent in some sector symmetrical to, and containing an infinite portion of, the positive real axis.

When $P_{n}$ is a constant, this follows quickly from $\$ 15$, for $f\left(e^{i \downarrow} z\right)$ satisfies all of the hypotheses of that article when $P_{n}$ is a constant.

When $P_{n}$ is not a constant, we make the substitution $P_{n} w=y$, whereupon (43) goes over into an equation in $y$ with unity for the coefficient of $y^{n}$. Thus $P_{n}\left(e^{i \psi} z\right) f\left(e^{i \psi} z\right)$ satisfies the hypotheses of $\$ 15$. Also the pair of functions $P_{n}\left(e^{i \psi} z\right) f\left(e^{i \psi} z\right)$ and $P_{n}\left(e^{i \psi} z\right)$ satisfy the hypotheses of $\$ 16$, so that we have our result for $f\left(e^{i \psi z}\right)$.

Let the expansion of $f\left(e^{i \psi} z\right)$ be normally convergent in a sector symmetric 
to the positive real axis, of opening $2 \delta_{\psi}<2 \pi$. By $\$ 17$, for $|\eta|<\delta_{\psi}$, we can get the expansion (25) of $f\left(e^{i(\psi+\eta)} z\right)$ from that of $f\left(e^{i \psi} z\right)$ by replacing $z$ by $e^{i \eta} z$. (Evidently the result of $\$ 17$ holds, for decreasing $\mu$ 's, when $O A$ has a negative inclination greater than $-\pi$.)

Consider the intervals $\left(\psi-\delta_{\psi}, \psi+\delta_{\psi}\right)$ and pick out a finite number of them whose interiors cover the entire interval $\left(\phi_{1}, \phi_{2}\right)$.

We see now that, for every $\psi$, the expansion of $f\left(e^{i \psi z}\right)$ can be found from that of $f\left(e^{i \phi_{1} z}\right)$ by replacing $z$ by $e^{i\left(\psi-\phi_{1}\right)} z$; for we can pass from $\phi_{1}$ to $\psi$ across the small intervals, in a finite number of steps.

We now consider the expansion (25) of $f\left(e^{i \phi_{1} z}\right)$ as a double series and order its terms so that the moduli of the exponents are non-decreasing. We have thus

$$
f\left(e^{i \phi_{1} z}\right)=v_{1} e^{\tau_{1} z}+\cdots+v_{p} e^{\tau_{p} z}+\cdots,
$$

where the $\tau$ 's are real or complex numbers such that $\left|\tau_{p}\right| \rightarrow \infty$ as $p \rightarrow \infty$.

We have from (45)

$$
f(z)=v_{1} e^{\sigma_{1} z}+\cdots+v_{p} e^{\sigma_{p} z}+\cdots,
$$

where $\sigma_{p}=e^{-i \phi_{1}} \tau_{p}$.

It is now easy to see that the second member of (46) converges normally to $f(z)$ in a subsector of $S$.

For the expansion (25) of $f\left(e^{i \psi_{z}}\right), \dot{\phi}_{1} \leqq \psi \leqq \phi_{2}$, is obtained from (46) by replacing $z$ by $e^{i \psi_{z}}$. Now as the expansion (25) of $f\left(e^{i \psi_{z}}\right)$ has $a$ 's which are exponential polynomials, the distant terms of (46) give distant terms in the expansion of $f\left(e^{i \psi z}\right)$. Hence, for every $\psi,(46)$ is normally convergent in a sector symmetrical with respect to the line amp $z=\psi$. The Heine-Borel theorem does the rest.

Furthermore, $f(z)$ can have only one expansion (46) normally convergent in a sector contained in $S$. For the proof, we assume, multiplying $z$ by a constant of modulus unity, if necessary, that the sector contains an infinite portion of the positive real axis. Then the normal convergence of (46) implies that the real part of $\sigma_{p}$ approaches $-\infty$ as $p$ increases. Hence, grouping finite sets of terms in (46), we can form a normally convergent series (25), which, of course, is unique. This shows that (46) is unique.

Let us see now what happens under the assumption that $f(z)$ is analytic throughout $S$, but not analytic throughout any sector containing $S$, with the same vertex as $S$ and of greater opening than $S$.

There will be a subsector of $S$ in which (46) converges absolutely. For (46) is obtained from the expansion (25) of $f\left(e^{i \phi_{1} z}\right)$ by replacing $z$ by $e^{-i \phi_{1} z}$, and (46) is obtained similarly from $f\left(e^{i \phi_{2} z}\right)$. 
On the other hand, in the expansion (25) of $f\left(e^{i \phi_{1} z}\right)$ (the $\mu$ 's of the expansion will increase) there must be an $a(z)$ which contains an infinite number of terms. For a series (25) with all $a$ 's exponential polynomials, found by the Newton polygon procedure from (43), could be considered either as having increasing $\mu$ 's or decreasing $\mu$ 's. It would therefore converge normally in a sector above, and in a sector below, the positive real axis; thus, by (b) of $\$ 15$, in a sector symmetric to the positive real axis. Hence $f(z)$ would be analytic in a sector containing $S$ and of wider opening than $S$.

Thus some term in the expansion of $f\left(e^{i \phi_{1} z}\right)$ will give an infinite number of terms of (46). There will be an $h>0$ such that an infinite number of terms of (46) exceed $e^{-h|z|}$ in modulus as $z$ increases with amp $z=\phi_{1}$. A similar state of affairs holds for amp $z=\phi_{2}$. Hence (46) is not normally convergent in any subsector of $S$.

20. Suppose that some branch $f(z)$ of a solution of (43) is analytic in a sector of opening greater than $\pi$. According to Hille, the domain in which (46) converges absolutely is convex, and (46) converges uniformly in any closed region interior to its domain of absolute convergence. (This is essentially (b) of $\$ 15$ and the result of $\$ 11$.)

Hence the expansion (46) of $f(z)$ will converge absolutely for every $z$, and uniformly in every finite area. Thus $f(z)$ is an integral function.

We shall prove that $f(z)$ is an exponential polynomial.

Since, in every sector of opening less than $2 \pi$, there is a subsector in which $f(z)$ has a normally convergent expansion (46), and since (46) is unique for any sector, $f(z)$ has a development (46) normally convergent all over the plane, that is, in a sector of opening $2 \pi$. Thus, the remainder after a large number of terms in (46) approaches zero uniformly all over the plane. As every remainder is an integral function, the remainder after a large number of terms is a constant, which has to be zero, for no $v e^{\sigma z}$ with $\sigma \neq 0$ and $v \neq 0$ can be a constant.

Thus $f(z)$ is an exponential polynomial. We shall prove that every exponent in $f(z)$ is a linear combination of the exponents of the $P$ 's in (43), with rational coefficients.

We have, identically in $w$ and $z$,

$$
P_{n} w^{n}+\cdots+P_{0}=(w-f(z))\left(P_{n} w^{n-1}+Q_{1} w^{n-2}+\cdots+Q_{n-1}\right),
$$

with every $Q$ an exponential polynomial.

From among the exponents present (effectively) in all of the $P$ 's, we select those whose real parts are a minimum, and from those just selected we pick that exponent which has a minimum coefficient for $(-1)^{1 / 2}$. Let $\beta$ be the exponent thus selected. We write (47) 


$$
e^{-\beta z} P_{n} w^{n}+\cdots+e^{-\beta z} P_{0}=(w-f(z))\left(e^{-\beta z} P_{n} w^{n-1}+\cdots+e^{-\beta z} Q_{n-1}\right) .
$$

In the functions $e^{-\beta_{2}} P_{i}$, those exponents which are not zero have either a positive real part or a zero real part and a positive coefficient for $(-1)^{1 / 2}$. Also zero is actually present as an exponent in certain of the functions.

If $w$ is given a constant value, the first member of (48) becomes an exponential polynomial in $z$. We attribute to $w$ such a constant value that the first member of (48) and also $w-f(z)$, when written as exponential polynomials, may each contain a constant term, that is, a term of exponent zero. This is clearly possible.

In $w-f(z)$, with $w$ the constant taken above, we pick out those exponents whose real parts are a minimum, and from the exponents just selected we choose that one for which the coefficient of $(-1)^{1 / 2}$ is a minimum. Let $\gamma$ be the exponent thus found. We write

$$
w-f(z)=e^{\gamma z}\left(e^{-\gamma z}(w-f(z))\right) .
$$

By the first theorem of our paper* $A$ factorization theory for functions $\sum a_{i} e^{\alpha_{i} x}$ every exponent in $e^{-\gamma z}(w-f(z))$ is linear in the exponents in the first member of (48) with rational coefficients. But since the exponent zero is present in $w-f(z)$, the exponent $-\gamma$ is present in $e^{-\gamma z}(w-f(z))$. Hence $\gamma$ is linear in the exponents of the first member of (48), with rational coefficients. Then every exponent in $w-f(z)$ is linear in the exponents in the functions $e^{-\beta_{z}} P_{i}$, with rational coefficients, and from what we know of $\beta$, this gives our result.

The results of the present article are given by the following two theorems:

THEOREM I. If a solution $w$ of (1) is a uniform function, more generally a function uniform in a sector of opening greater than $\pi$, then $w=Q / P_{n}$, where $Q$ is an exponential polynomial whose exponents are linear combinations of the exponents in the P's, with rational coefficients.

THEOREM II. If $P / Q$, with $P$ and $Q$ exponential polynomials, is analytic in a sector of opening greater than $\pi$, then $P / Q$ is an exponential polynomial. $\dagger$

21. We write (43)

$$
w^{n}+Q_{n-1} w^{n-1}+\cdots+Q_{0}=0,
$$

where $Q_{i}=P_{i} / P_{n}$. We shall call (49) irreducible if its first member is not the product of two polynomials in $w$, both of degree less than $n$, with coefficients which are the quotients of two exponential polynomials.

* Ritt, (2).

† Cf. Ritt, (1). [Remark inserted in proof. The method of (1) can be extended so as to yield Theorem II.] 
Theorem III. An irreducible equation (49) defines only a single analytic function.

Consider any area in which the $n$ solutions of (49) are analytic.

Suppose that $w_{1}, \cdots, w_{r}, r<n$, are distinct solutions of (49) which are continuations of each other, but of no other solution of (49). Then the symmetric functions of $w_{1}, \cdots, w_{r}$ are uniform all over the plane. Since the symmetric functions satisfy equations like (49), the symmetric functions are quotients of exponential polynomials. This shows that (49) is not irreducible.

We prove now

THEOREM IV. Let (49) be irreducible, with $n>1$. Let $S$ be any sector of opening greater than $\pi$. Then there exist in $S$, and outside an arbitrarily large circle, branch points of which generate the entire group of monodromy of $w$.

Suppose that those branch points which lie in $S$, and outside some circle, generate only a proper subgroup $H$ of the group of monodromy, $G$. Construct a rational combination $R$ of the solutions of (49) which is invariant (as a function of $z$ ) under $H$, but not under $G$. Then $R$ is uniform in $S$. As $R$ is a solution of an equation like (49), $R$ is uniform all over the plane, hence invariant under $G$. This contradiction proves our theorem.

22. We consider an equation (43) for which the corresponding equation (49) is irreducible. A ray emanating from the origin will be called singular if there does not exist a sector, containing in its interior an infinite portion of the ray, in which all of the $n$ branches of $w$ are analytic.

We shall prove that $w$ has at most a finite number of singular rays.

Let $\phi$ be any angle. Then, by section III, the $n$ solutions of

$$
P_{n}\left(e^{i \phi_{z}}\right) w^{n}+\cdots+P_{0}\left(e^{i \phi_{z}} z\right)=0
$$

are analytic in two sectors, one below, one above the real axis, each with a side in the direction of the positive real axis. Then the ray $\operatorname{amp} z=\phi$ cannot have singular rays making arbitrarily small angles with it. This proves that there are at most a finite number of singular rays.

According to Theorem II, if $w$ is not an integral function, every sector with vertex at the origin, of opening greater than $\pi$, contains at least one singular ray. Hence, if $w$ is not an integral function, it has at least two singular rays. When there are just two singular rays, the angle between them is $\pi$.

If $w$ is not uniform, then no branch of $w$ can be uniform in any sector of opening greater than $\pi$. In that case, $w$ must have at least two singular rays such that, in any sector containing one of them, there are an infinite number of branch points of $w$. Singular rays of this type will be called branch rays.

Let $w$ have $r \geqq 2$ singular rays, which divide the plane into sectors 
$S_{1}, \cdots, S_{r}$. It is easy to see that in every $S_{i}$ there is a subsector in which every branch of $w$ is analytic. According to $\$ 19$, every branch of $w$ has a development (46), absolutely convergent in some subsector of $S_{i}$. If $T_{i}$ is any sector with vertex at the origin every point of which, except the vertex, is interior to $S_{i}$, the development (46) of every branch is normally convergent in a subsector of $T_{i}$.

It will be shown later by examples that some of the branches of $w$ may be analytic in a sector containing in its interior an infinite portion of a singular ray of $w$.

We summarize the principal results of $\$ \S 19,21$ in the following theorem, in which it is understood that every $Q$ is the quotient of two exponential polynomials.

THEOREM V. Let $w$ be the solution of the irreducible equation

$$
w^{n}+Q_{n-1} w^{n-1}+\cdots+Q_{0}=0 .
$$

Then $w$ has a finite number of singular rays. Given any two adjacent singular rays, there is a subsector of the sector formed by them in which every branch of $w$ is analytic. If $w$ is not integral, it has at least two singular rays. If $n>1$, $w$ has at least two branch rays, and no branch of $w$ is uniform in a sector of opening greater than $\pi$. If a branch $w_{1}$ is analytic in a subsector of the sector $S$ formed by two (not necessarily adjacent) singular rays, then w can be expanded in a series

$$
v_{1} e^{\sigma_{1}^{z}}+\cdots+v_{p} e^{\sigma_{p 2}}+\cdots ; \lim \left|\sigma_{p}\right|=\infty,
$$

with constant v's, absolutely convergent in some subsector $T$ of $S$. In any sector with the same vertex as $T$, and lying, except for its vertex, interior to $T$, the expansion is normally convergent. There is but one expansion of this type which converges normally to $w_{1}$ in a sector contained in $S$. If $w_{1}$ is not analytic in any sector with the same vertex as $T$, and containing $T$, except for the vertex, in its interior, the expansion is not normally convergent throughout $T$.

23. The singular rays of $w$ admit of quite precise description in terms of the exponents in the $P$ 's of equation (1).

The poles of $w$ are all located at the zeros of $P_{n}$. Those branch points of $w$ which are not zeros of $P_{n}$ are found by equating the discriminant of (1) to zero. The discriminant is a polynomial in the $P$ 's divided by a power of $P_{n}$. Hence the singular points of $w$ are found by equating to zero two exponential polynomials, $P_{n}$ and the numerator of the discriminant.

The distribution of the zeros of exponential polynomials has been investigated by Tamarkin, Wilder, and Pólya in papers to which reference 
has already been made. Of their results, the following is the one of chief interest for us.

Consider any exponential polynomial $P$. Let the exponents in $P$ be plotted in the complex plane, and let the smallest convex polygon $\mathfrak{A}$ which contains the exponents, in its interior or upon its boundary, be constructed. Let the sides of $\mathfrak{A}$ be $\sigma_{1}, \cdots, \sigma_{p}$. Let $d_{i}$ be a ray which is the image, with respect to the real axis, of the normal to $\sigma_{i}$ directed outwardly to $\mathfrak{A}$. Then there exist $p$ half-strips, each parallel to, and directed like, some ray $d_{i}$, which contain all of the zeros of $P$. If the length of $\sigma_{i}$ is $s_{i}$, the number of zeros in the strip parallel to $d_{i}$ and less than $r$ in modulus is equal to

$$
\frac{\sigma_{i} r}{2 \pi}+O(1)
$$

The singular rays of $w$ are parallel to the rays $d_{i}$, constructed for $P_{n}$ and for the numerator of the discriminant. Of course, not every $d_{i}$ need give a singular ray.

We shall show by means of an example that, along the distant part of a branch ray, the branches of $w$ may break up into sets such that branches belonging to different sets are not connected by those branch points of $w$ which cluster about the given branch ray. For instance, $w$ may have a branch which is uniform in a sector containing an infinite portion of a branch ray of $w$.

Let $w$ be defined by

$$
w^{3}+3 e^{2} w^{2}+e^{(2+i) z} w+1=0 .
$$

The exponents in the discriminant of $w$ are found to be $6+2 i, 6+3 i, 0,3$ and $3+i$. The rays $d_{i}$ for the discriminant are found to have inclinations 0 , $\pi / 2, \arctan 3 / 2$ (first quadrant), arctan 2 (third quadrant). When we seek the expansions (25), according to the method of III, for the three branches of $w$, we find one expansion with $a$ 's which are exponential polynomials, and two with $a$ 's which are infinite series. This means that one branch of $w$ is uniform along the distant part of the positive real axis, while two branches are connected with each other by branch points of arbitrarily large modulus in a half-strip directed like the positive real axis.

We find a similar state of affairs along the ray of slope $3 / 2$, which is investigated by making the substitution $z=(2+3 i) z^{\prime}$. There is one uniform branch and the other two are connected.

Along the rays of slopes $\infty$ and 2, all of the branches are connected.

The following example shows that $w$ may have an infinite number of poles along a singular ray and that still some branch of $w$ may be analytic in a sector containing the distant part of the ray. Let $w$ be defined by 


$$
\left(e^{i z}-1\right) w^{2}-2 e^{z} w-1=0 .
$$

Then $w$ has a pole for every integral value of $z$. Still one of the developments (25) of $w$ has $a$ 's which are exponential polynomials. This is seen on writing

$$
w=\frac{e^{z} \pm\left(e^{2 z}+\left(e^{i z}-1\right)\right)^{1 / 2}}{e^{i z}-1}
$$

and expanding the radical in powers of $\left(e^{i z}-1\right) / e^{2 z}$. Thus one of the branches of $w$ is analytic in a sector containing an infinite portion of the positive real axis.

24. Let us imagine that some branch $f(z)$ of $w$ is analytic in a half-plane, to fix our ideas, in an upper half-plane. Then the development (25) of $f(-z)$, with decreasing $\mu$ 's, is found from that of $f(z)$ with increasing $\mu$ 's by replacing $z$ by $-z$. As both developments have decreasing $\lambda$ 's, both developments have only a finite number of $\lambda$ 's. Hence $f(z)$ is of the form

$$
a_{1}(z) e^{\lambda_{1} z}+\cdots+a_{p}(z) e^{\lambda_{p} z}
$$

where each $a(z)$ is an algebraic combination of exponentials with pure imaginary exponents. It is evident that (51) will represent all of the branches of $w$, all over the plane. Hence we can remove a strip from the plane and leave two half-planes in which every branch of $w$ is analytic.

Consider a $w$ which has just three branch rays. From what precedes, we see that no two of them are extensions of each other. It follows from Theorem IV that the branch points which cluster about the distant portion of any branch ray connect up all of the branches of $w$.

\section{REFERENCES}

Hille, Note on Dirichlet series with complex exponents, Annals of Mathematics, (2), vol. 25 (1924), pp. 261-278.

P6lya, Geometrisches ueber die Verteilung der Nullstellen gewisser ganzer transzendenter Funktionen, Münchener Berichte, 1920.

Ritt, (1) On the zeros of exponential polynomials, these Transactions, vol. 31 (1929), pp. 680-686.

(2) A factorization theory for functions $\sum_{i=1}^{n} a_{i} e^{\alpha_{i} x}$, these Transactions, vol. 29(1927), pp. 584-596.

Tamarkin, Some general problems in the theory of ordinary linear differential equations and expansion of an arbitrary function in series of fundamental functions, Mathematische Zeitschrift, vol. 27 (1928), pp. 1-54. Also, earlier papers there mentioned.

Schwengler, Geometrisches ueber die Verteilung der Nullstellen spezieller ganzer Funktionen, Dissertation, Zurich, 1925.

Wilder, Expansion problems in ordinasy linear differential equations with auxiliary conditions at more than two points, these Transactions, vol. 18 (1917), pp. 415-432.

Columbia UNIVERSITY, NEW YoRK, N. Y. 